\title{
Whole-body Parametric Imaging of FDG PET using uEXPLORER with Reduced Scan Time
}

Running title: Fast total-body PET Ki imaging

Authors: Yaping $\mathrm{Wu}^{1}$, Tao Feng ${ }^{2}$, Yizhang Zhao ${ }^{3}$, Tianyi Xu${ }^{3}$, Fangfang $\mathrm{Fu}^{1}$, Zhun Huang ${ }^{1}$, Nan Meng ${ }^{1} \mathrm{Hongdi}^{2}{ }^{2}$, Fengmin Shao ${ }^{1}$, Meiyun Wang ${ }^{1}$

\section{Affiliations:}

${ }^{1}$ Department of Medical Imaging, Henan Provincial People's Hospital, China

${ }^{2}$ UIH America Inc. TX, USA

${ }^{3}$ United Imaging Healthcare, Shanghai, China

First author: Tao Feng and Yaping Wu contributes equally to this work. Yaping Wu: Department of Medical Imaging, Henan Provincial People's Hospital, 7 Weiwu Road, Zhengzhou, 450003, Henan, China, E-main: ypwu@ha.edu.cn Phone: +86-15838178262, Tao Feng: UIH America, Inc. 9230 Kirby Dr, Suite 600, Houston, TX, 77054 USA E-mail: tao.feng@united-imaging.com, Phone: +1(443)927-6712, Tax: (281)709-6856.

Corresponding author: Meiyun Wang: Department of Medical Imaging, Henan Provincial People's Hospital, 7 Weiwu Road, Zhengzhou, 450003, Henan, China, E-main: mywang@ @ha.edu.cn Phone: +86-037165580267

Word count: 4991

Figures: 7

Tables: 2

Financial support: This work was partially supported by National Key R\&D Program of China (2017YFE0103600), National Science Foundation of China (81720108021), Zhongyuan Thousand Talents Plan Project (ZYQ201810117), and Zhengzhou Collaborative Innovation Major Project (20XTZX05015) 
Parametric imaging of $K_{i}$ (the net influx rate) in FDG PET has been shown to provide better quantification and improved specificity for cancer detection compared with SUV imaging. Current methods for generating parametric images usually requires a long dynamic scan time. With the recently developed UEXPLORER scanner, a dramatic increase of sensitivity has reduced the noise in dynamic imaging, making it more robust to employ a non-linear estimation method and flexible protocols. In this work, we explored 2 new possible protocols besides the standard 60-minute one for the possibility of reducing scan time for $K_{i}$ imaging.

Method:

In the proposed protocols, the first one is the conventional dynamic scans of a 60-minute scan time as the gold standard. The second scanning protocol includes two scan periods, 0-4 minutes, and 54-60 minutes post-injection. The third one consisted of a single scanning period from 50 minutes to 60 minutes post-injection. In the third protocol, a second injection was applied at $\mathrm{t}=56 \mathrm{minutes}$. The two new protocols were simulated from the 60-minute scans. A hybrid input function that combining the population-based input function and the image-derived input function (IDIF) was used. The results were also compared with the IDIF acquired from Protocol 1. A previously developed maximum likelihood approach was used for estimating the $K_{i}$ images. A total of 7 cancer patients scanned using the uEXPLORER scanner were enrolled in this study. Lesions were identified from the patient data and the lesion $K_{i}$ values were compared among different protocols.

Results:

The acquired hybrid input function was comparable in shape with the IDIF for each patient. The average area-under-curve difference was $\sim 3 \%$, suggesting good quantitative accuracy. The visual difference between the $K_{i}$ images generated using IDIF and the hybrid input function was also minimal. The acquired $K_{i}$ images using different protocols were visually comparable. The average $K_{i}$ difference in the lesions was found to be $2.8 \% \pm 2.1 \%$ for protocol 2 and $1 \% \pm 2.2 \%$ for protocol 3 .

Conclusion:

The results suggest that it is possible to acquire the $K_{i}$ image using the nonlinear estimation approach with much-reduced scan time. Among the two new protocols, the protocol with dual injections shows the greatest promise in terms of practicality. 


\section{INTRODUCTION}

Positron Emission Tomography (PET) with the standard uptake values (SUV) $(1,2)$ is widely used in clinical oncology for tumor imaging. However, the use of SUV suffers from several drawbacks (3). For instance, the uptake time of FDG in different tumors may vary significantly in kinetics for different tissue (4). It is also challenging for the SUV measurement to differentiate malignant tumors from processes such as inflammation (5-7).

Parametric imaging provides an alternative to SUV imaging and it has the potential for added information. For FDG studies, a few parameters are commonly derived, such as the net influx rate $K_{i}$, the delivery rate constant $K_{l}$, and blood fractions in tissue. $K_{i}$ is more commonly used and often acquired using Graphical methods due to its simplicity (8). The acquired $K_{i}$ has been found to yield improved specificity at a similar sensitivity for cancer detections (9). It has also been found that the $K_{i}$ image yielded better results for tumor volume delineation than the SUV image (10). FDG $K_{1}$ alone or with the combination of $K_{i}$ was found to be an indicator for identifying tumor subgroups (11), evaluation of chemotherapy response (12). The combined FDG parameters were found to be helpful for accessing metabolic tumors (13) as well.

Compared with SUV imaging, parametric imaging also has its challenges. One is the need for an accurate input function. The conventional approach utilizes the invasive use of arterial samples. In recent years, more and more studies are suggesting that the image-derived input function $(14,15)$, population-based input function (16), or hybrid input function with both image data and population samples (17) can be used as the non-invasive replacement. Another practical issue is the much-increased scan time. To estimate the net influx rate $K_{i}$ using the conventional Patlak method, a much longer scan is unavoidable. This is because the $K_{i}$ image is the slope image in the Patlak model, with slow-changing dynamics, it requires a long scan time to accurately estimate the change of activity. As a result, a minimal scan time of 30 minutes is often used for estimating $K_{i}$ with the Patlak model. Compared with the state-of-art whole-body SUV scans that last less than 10 minutes, the much-increased scan time has limited the daily application of parametric imaging. The much-increased scan time also increases the likelihood of patient motion during scans, which may further degrade image quality.

While conventionally, single bed acquisition is usually used for parametric imaging, whole-body Patlak analysis using regular scanners (18) or the total-body uEXPLORER scanner (19) have recently been proposed and validated, 
Whole body parametric imaging provides a unique opportunity for the inspection of disseminated disease, which is also a major application of PET imaging.

Compared with the Graphical method, $K_{i}$ could also be estimated using a non-linear approach with the use of a 2-tissue irreversible compartment model. An entire time-activity curve (TAC) consisted of 60 minutes scan or even longer scan time is usually used for this purpose. With the UEXPLORER (20), the much-increased sensitivity for the whole-body scan has dramatically reduced the noise in the reconstructed dynamic images. The reduction of noise in the dynamic images has made non-linear estimation more robust. An advantage of non-linear estimation is that it can better utilize dynamic data other than that after equilibrium, unlike conventional linear Patlak models (21), therefore providing more freedom in protocol designs. In previous studies, we have demonstrated the possibility of reduced scan time for estimating parameters such as $K_{l}$ and the blood fraction (22). In this work, we further explored the possibility of accurately estimating $K_{i}$ using a much shorter dynamic scan sequence with a total scan time of 10 minutes for whole-body imaging. Two alternatives were investigated. One utilizes the combination of early time point and late time point scan (dual points scan), the other uses the dual-injection protocol to combine both early dynamic information and late dynamic information within one scan duration.

\section{METHODS}

\section{A. Scanning protocols}

Three protocols were proposed and studied in this paper. The first protocol was the regular 0-60 minutes dynamic scan. It was used as the gold standard to evaluate the performance of the other protocols.

The second protocol consisted of the combination of 2-time points, i.e. the early time point $(0-4$ minutes post-injection, p.i.) and the late time points (54-60 minutes p.i.). In this protocol, for the minimal use of scanner time, the patients are required to scan two times, and registration is required in between the scans. In this study, as a proof of concept, this protocol was simulated by excluding the 4-54 minutes p.i. of an entire 0-60 minutes p.i. dynamic scan range without the need for registration. The main goal of this protocol was to examine the accuracy of the estimation by using only the early and the late phase information. 
In the third scan protocol, a single scan period was used (50-60 minutes p.i.) with the help of dual injections scheme. The first injections occur in $\mathrm{t}=0$, and the second injections occur in $\mathrm{t}=56$ minutes p.i. In this case, the last 4 minutes provides the early dynamic data, and the first 6 minutes provides similar information contained in the second scanning period in Protocol 2. This scanning protocol was simulated by combining the dynamics images of the 0-4 minutes p.i. and 56-60 minutes p.i. Figure 1 shows an illustration of the three protocols. Table 1 shows the dynamic time frame of all the protocols.

\section{B. Input functions}

For the protocol using the entire 60 minutes, the image-derived input function (IDIF) is used. Ascending aorta was used to extract IDIF as it is less affected by respiratory motion. For the other scans, with the limited scan time, IDIF was not available for the entire dynamic range. In this paper, we acquired the input function using a hybrid approach by combining the population-based, the model-based, and the IDIF. The input function for the second scanning protocol is proposed as:

$$
C_{p}(t) \triangleq\left\{\begin{array}{lr}
C_{\text {image } 1}(t) & t \leq t_{1} \\
\mu e^{-\gamma\left(t-t_{1}\right)} C_{p 0}(t) & t \geq t_{1} \text { and } t \leq t_{2} \\
C_{\text {image } 2}(t) & t \geq t_{2}
\end{array}\right.
$$

where $C_{\text {image } 1}(t)$ is the IDIF of the first 4 minutes, $C_{\text {image } 2}(t)$ is the IDIF on the last 6 minutes. $C_{p 0}(t)$ is the population-based input function, $\gamma$ and $\mu$ is the scaling constant that satisfy $\mu C_{p 0}\left(t_{1}\right)=C_{\text {image } 1}\left(t_{1}\right)$, and $\mu e^{-\gamma\left(t_{2}-t_{1}\right)} C_{p 0}\left(t_{2}\right)=C_{\text {image } 2}\left(t_{2}\right)$.

For the third scanning protocol. The input function was acquired based on two assumptions: 1, the later phase of the input function can be approximated as a single exponential function. 2, the shape of the input function from the second injection has the same shape as the input function from the first injection. In literature studies with multiple injections on one patient, the similarity of the produced input function (23) supports the second assumption. 
The IDIF was first separated into two regions: before the second injection $\left(C_{B}(t)\right)$ and after the second injection $\left(C_{A}(t)\right)$, where $t_{0}$ represents the second injection time. An exponential curve $\left(C_{B 0} e^{-b t}\right)$ was used to fit $C_{B}(t)$ and the contribution of the second injection was estimated by subtracting the exponential curve from the IDIF $C_{A}(t)$, i.e. $C_{A}(t)-C_{B 0} e^{-b t}$. The contribution from the second injection was treated as the early phase input function $\left(C_{i m a g e 1}(t)\right)$ as that in Protocol 2, and $C_{B}(t)$ was treated as the late phase input function $\left(C_{\text {image }}(t)\right)$ as that in Protocol 2. The missing part was approximated using the same approach as shown in Equation (1).

For both the second and the third scanning protocol, the original IDIF acquired using the whole dynamic process was used as the gold standard.

\section{C.Maximum-likelihood estimation}

The dynamic changes of FDG within the human body can be approximated using the 2-tissue compartment model, where the first compartment $\left(C_{1}\right)$ describes the perfusion of FDG to the tissue, and the second compartment $\left(C_{2}\right)$ models the phosphorylation process within the cells. The two compartments can be modeled mathematically using the rate constants:

$$
\left\{\begin{array}{c}
\frac{d C_{1}}{d t}=K_{1} C_{p}+k_{4} C_{2}-k_{2} C_{1}-k_{3} C_{1} \\
\frac{d C_{2}}{d t}=k_{3} C_{1}-k_{4} C_{2}
\end{array}\right.
$$

where $K_{1}$ and $k_{2}$ describe the forward and backward perfusion process of FDG in the tissue. $k_{3}$ and $k_{4}$ describe the phosphorylation and dephosphorylation process. In many cancer cells, the FDG-6-phosphate is only minimally dephosphorylated and is trapped within the cell (24). This process allows us to simplify the model by assigning $k_{4}=0$. The acquired dynamic PET image, $X(t)$, can be represented using the equation below when $k_{4}=0$.

$$
X=v_{b} C_{p}+C_{1}+C_{2}=v_{b} C_{p}+\frac{k_{2} K_{1}}{k_{2}+k_{3}} \exp \left(-\left(k_{2}+k_{3}\right) t\right) \otimes C_{p}(t)+\frac{k_{3} K_{1}}{k_{2}+k_{3}} \int C_{p} d t
$$


In the above equation, define $K_{1}^{\prime}=K_{1} \frac{k_{2} K_{1}}{k_{2}+k_{3}}, k_{2}{ }^{\prime}=k_{2}+k_{3}, K_{i}=\frac{k_{3} K_{1}}{k_{2}+k_{3}}, C_{i}(t)=\int C_{p} d t$, the above equation can be written as:

$$
X=v_{b} C_{p}+C_{1}+C_{2}=v_{b} C_{p}(t)+K_{1}{ }^{\prime} \exp \left(-k_{2}{ }^{\prime} t\right) \otimes C_{p}(t)+K_{i} C_{i}(t)
$$

The above equation is similar to the Patlak model, where the $K_{i}$ has the same definition as that in the Patlak model, and the combined effect of $v_{b}+K_{1}{ }^{\prime} \exp \left(-k_{2}{ }^{\prime} t\right) \otimes / C_{b}(t)$ was treated as a constant after equilibrium in the Patlak model.

With the assumption that the voxel values in the dynamics image approximately follow a scaled Poisson distribution (25), the maximum-likelihood estimation approach was used for estimating $K_{i}$. The update equation for $K_{i}$ can be derived as:

$$
K_{i}^{p+1}=\frac{K_{i}^{p}}{\sum_{t} C_{i}(t)} \sum_{t} \frac{C_{i}(t) \boldsymbol{X}(t)}{\widehat{\boldsymbol{C}}^{p}(t)}
$$

where $\widehat{\boldsymbol{C}}^{p}(t)=v_{b}^{p} C_{b}+{K^{\prime}}_{1}^{\prime p} e^{-k_{2}^{\prime} t} \otimes C_{p}(t)+{K^{\prime}}_{i}^{p} C_{i}(t)$ is the estimated dynamic image at time $\mathrm{t}$ given the estimated parametric images, $p$ is the iteration number. The effects of different frame lengths were included in $C_{p}$ and $C_{i}$ in the above equations. The derivation is similar to our previous proposed update equation for $v_{b}$ and $K_{1}$ (22). For whole-body imaging, the input function $C_{p}$ may also be subject to the delay and dispersion effects. The delay effect is modeled and estimated using the same approach proposed in (22). A total of 5 parameters, including $K_{1}{ }^{\prime}, k_{2}{ }^{\prime}, v_{b}, K_{i}$, and the time delay, were estimated jointly. The estimated $K_{i}$ was analyzed subsequently as it is the target of interest in this study.

D. Patient data and image reconstruction 
In our study, seven potential cancer patients (Table 2) referred to the Henan Hospital were acquired using the uEXPLORER scanner (Shanghai United Imaging Healthcare, China) with the dynamic scanning protocol. The patient group was preselected to exclude those with significant motion artifacts, and those with non-bolus input functions. Visual examinations were used to determine the motion artifacts, and the exclusion criteria were those with visible motion larger than 5 voxels or $15 \mathrm{~mm}$. The dynamic study has been approved by the Institutional Review Board of the hospital, and a written informed consent form has been obtained from each patient. The patient data is consisted of 4 males and 3 females, with weight being $66 \pm 13 \mathrm{~kg}$, and injected dose as $273 \pm 60 \mathrm{MBq}$. The leg was chosen as the injection site as it is closer to the end of the gantry.

Dynamic image reconstruction was performed using the vendor recommended settings with random, scatter, attenuation, normalization, and deadtime corrections, the reconstructed image has 2.89-mm slice thickness and 3.125-mm voxel size in the transaxial plane. The number of voxels in the reconstructed image is 192 by 192 by 672 . Time-of-flight (TOF) reconstructions have been applied using manufacturer-supplied reconstruction software (OSEM with 3 iterations and 24 subsets) with the point-spread-function (PSF) model.

An alternate update approach was applied for the joint estimation process. A total of 27 main iterations were used, where in each iteration, 6 sub-iterations were used for $K_{i}, K_{l}$, , the blood fraction (with total iteration number of 162), 2 sub-iterations were used for the time delay, and 1 sub-iterations were used for $k_{2}{ }^{\prime}$. The $K_{i}$ estimated using the first protocol with the IDIF was selected as the gold standard. $K_{i}$ was also estimated using the conventional Patlak model for comparison. In the Patlak method, data from 20 minutes p.i. to 60 minutes p.i. with IDIF was used. The framing sequence is the same as Protocol 1. No post-smoothing filter was applied. The noise of the estimated images was calculated using VOIs in the thigh muscle region. The coefficient of variation was used as the surrogate for noise. A region growing approach with a threshold being $90 \%$ of the maximum value in the $K_{i}$ image (Protocol 1 ) was used for the acquisition of the lesions. The average $K_{i}$ value of the lesions from images acquired using different methods was also measured to study the quantitative accuracy.

\section{RESUltS}


Figure 2 shows the input functions acquired from a patient using the IDIF approach and the proposed hybrid approach. The original population-based input function (17) was also displayed with normalized peak value. A significant difference existed between the population-based and the IDIF when normalized to the same peak value, but a good agreement can be achieved with the hybrid method. For all patient data, the average area-under-curve ratio between the hybrid input function and the IDIF was $1.03 \pm 0.04$, suggesting it is possible to use the hybrid input function for $K_{i}$ estimation in cases where the whole dynamic data is unavailable.

Figure 3 shows the reconstructed $K_{i}$ images of Patient 1 using the three protocols $\left(K_{l}\right.$ ' and $k_{2}{ }^{\prime}$ images were included in Supplemental Figure 1 and Figure 2). The same color scale was used for all the images. The IDIF were used in the first protocol and both IDIF and hybrid input function was used for the other protocols. The $K_{i}$ images acquired using the second and the third protocol were visually comparable to that using Protocol 1 but noisier. Figure 4 shows the difference images of $K_{i}$ generated using different protocols (supplemental Figure 3 shows the corresponding percentage images). Minimal difference was observed between IDIF-based and hybrid input function generated $K_{i}$ images. Figure 5 shows the maximum intensity projections of the reconstructed $K_{i}$ images as well as the SUV image for Patient 5. The same scale was used for all $K_{i}$ images and the SUV image was scaled for comparable muscle uptakes.

The image noise using the three estimations were also calculated. The average coefficient of variation was $0.12 \pm 0.04$ for the $K_{i}$ images estimated using Protocol 1 in the thigh muscle region, $0.22 \pm 0.05$ for the $K_{i}$ images estimated using Protocol 2, and 0.20 \pm 0.04 with Protocol 3. The much-reduced noise level in Protocol 1 is likely caused by the long scan time. Protocol 3 also shows reduced noise level when compared with Protocol 2, which is likely caused by the use of summed data, as it is effectively 2 times the dose compared with Protocol 2.

Using the first protocol, $K_{i}$ was calculated with the proposed non-linear approach and the conventional Patlak approach (Figure 6). The images in general agree with each other with some minor differences. The noise level in the nonlinear estimation is visually lower than that of the linear estimation, which is in agreement with literature findings (26). A higher muscle background $K_{i}$ value was detected in the non-linear approach.

A total of 26 lesions were identified and segmented from the patients. The same ROI was used for different $K_{i}$ images generated in different protocols for consistency. The average diameter of the segmented lesions is $13.8 \mathrm{~mm}$. The $K_{i}$ 
values inside the ROI measured by the gold standard and the protocols with the reduced scan time is plotted in Figure 7. An example of the fitted TAC is included in supplemental Figure 4. The mean difference between the second protocol and the first protocol is $2.8 \%+-2.1 \%$. The mean difference between the third protocol and the first protocol is $1 \%+-2.2 \%$. This result suggests that with a total scan time of 10 minutes, the new protocols were able to maintain quantitative accuracy for the lesions despite the much-reduced scan time.

\section{DISCUSSION}

The $K_{i}$ difference between images (Figure 4C) estimated using IDIF and the hybrid input function was negligible in most cases, suggesting the hybrid input function can be a reliable approach for estimating the $K_{i}$ images. However, with the use of the hybrid input function, quantitative errors could still be present due to patient variations. The hybrid input function was also non-invasive and does not relies on data outside the acquisition period, making it easier to be incorporated in clinical studies.

Due to the residual activity around the injection site, some differences were present in the veins on the left leg among different protocols. A large difference of $K_{i}$ was observed in the kidney region among the different protocols. The difference could be explained by the fact that the 2-tissue irreversible model cannot accurately model the renal excretion process, and therefore a large difference could be expected with different protocols. In general, the $K_{i}$ images show much-improved lesion contrast when compared with the SUV image, suggesting improved clinical value with $K_{i}$ imaging.

While a good quantitative accuracy was observed in the lesions, a slight overestimation was observed in the muscle and liver region with the non-linear approach and Protocol 3. One reason could be the reduction of estimation accuracy due to combined early-phase and later-phase information in the dynamic data. As shown in the supplemental figures, the accuracy of estimated $K_{1}$ ' and $k_{2}$ ' in Protocol 3 was also not as good as that in Protocol 2. Another reason could be the model mismatch effect. As it was shown that the 2-tissue-irreversible may not be true in some tissue as non-zero $k_{4}$ can be expected in some normal cells (24) and therefore different estimated results could be expected with different estimation methods or protocols. 
A limitation of our approaches is that it requires 2 scanning sessions (Protocol 2) or 2 FDG administrations (Protocol

3). This makes the methods less practical, but it reduces the overall time spend on the PET/CT system. The second protocol requires additional image registration, which was not modeled in this study. The additional image registration may also introduce image artifacts that were not studied in this study. A second CT or low-dose CT scan may also be required in the second scan for accurate image registrations and attenuation correction. These challenges make Protocol 2 less practical. On the other hand, the estimation method in Protocol 2 provides a foundation for Protocol 3 to work, as it shows that $K_{i}$ can be estimated by combining the early phase and the late phase dynamic data. Protocol 3 provides a much better alternative for the practical application of fast $K_{i}$ imaging with dual injections, as the data were acquired in a single scan frame without the need for registration or another CT. The absence of a second scan also makes patient management much easier and reduces the likelihood of voluntary patient motion due to a much shorter scan time. However, patient motion could still impact the method and therefore motion compensation is still required for an improved quantitative result. There are challenges in the third protocol as well. One potential issue for Protocol 3 in this study is the assumption that the early phase input function is the same as the second bolus injection. Future studies are required to study the impact of this effect. The direct addition of the images for simulating Protocol 3 also doubles the effective injected dose, making the estimated noise in Protocol 3 smaller than that in Protocol 2. When keeping the injection dose the same, the image noise is expected to be higher when using Protocol 3.

Sluis et al, also showed the possibilities of reducing the scan duration using the conventional Patlak model with the help of the population-based input function (27). The advantage of using the Patlak model is that it only requires a single injection. With the dual injection protocols proposed in this study, we can achieve an even shorter scan time and the potential of multi-parametric imaging. Both approaches could be useful in clinical situations to promote the practical use of parametric imaging protocols.

\section{CONCLUSION}

In this study, we have shown that with the modified protocols, it is possible to dramatically reduce the required scan time for whole-body $K_{i}$ imaging to 10 minutes. The estimation of $K_{i}$ is possible due to the presence of both early-phase and late-phase information in the new protocols. The reduction of scan time makes it easier for incorporating $K_{i}$ imaging into routine clinics. 


\section{DISCLOSURES}

Tao Feng and Hongdi Li are employed by UIH America, Inc. Yizhang Zhao and Tianyi Xu are employed by United Imaging Healthcare. The other authors working with Henan Provincial People's Hospital have full control of the data and declare that they have no conflict of interest.

\section{KEY POINTS}

QUESTION: Can whole-body $K_{i}$ imaging be achieved using in a much-reduced scan time (10 minutes)?

PERTINENT FINDINGS: We have demonstrated with the new protocols (dual scan points or dual injections), it is possible to generate whole-body $K_{i}$ images with a total scan time for 10 minutes.

IMPLICATIONS FOR PATIENT CARE: A much reduced scan time for $K_{i}$ imaging improves the practicality for parametric imaging. A wider application of parametric imaging could be helpful for better diagnosis and treatment. 


\section{REFERENCES}

1. Delbeke D, Coleman RE, Guiberteau MJ, et al. Procedure guideline for tumor imaging with 18F-FDG PET/CT 1.0. J Nucl Med. 2006;47:885-895.

2. Lindholm P, Minn H, Leskinen-Kallio S, Bergman J, Ruotsalainen U, Joensuu H. Influence of the blood glucose concentration on FDG uptake in cancer-a PET study. J Nucl Med. 1993;34:1-6.

3. Adams MC, Turkington TG, Wilson JM, Wong TZ. A systematic review of the factors affecting accuracy of SUV measurements. Am J Roentgenol. 2010;195:310-320.

4. Shankar LK, Hoffman JM, Bacharach S, et al. Consensus recommendations for the use of 18F-FDG PET as an indicator of therapeutic response in patients in National Cancer Institute Trials. $J$ Nucl Med. 2006;47:1059-1066.

5. Metser U, Even-Sapir E. Increased 18F-fluorodeoxyglucose uptake in benign, nonphysiologic lesions found on whole-body positron emission tomography/computed tomography (PET/CT): accumulated data from four years of experience with PET/CT. In: Seminars in Nuclear Medicine. Vol 37. Elsevier; 2007:206-222.

6. Shreve PD, Anzai Y, Wahl RL. Pitfalls in oncologic diagnosis with FDG PET imaging: physiologic and benign variants. Radiographics. 1999;19:61-77.

7. Strauss LG. Fluorine-18 deoxyglucose and false-positive results: a major problem in the diagnostics of oncological patients. Eur J Nucl Med. 1996;23:1409-1415.

8. Patlak CS, Blasberg RG. Graphical evaluation of blood-to-brain transfer constants from multiple-time uptake data. Generalizations. J Cereb Blood Flow Metab. 1985;5:584-590.

9. Magri A, Krol A, Lee W, Lipson E, McGraw W, Feiglin D. A new method to determine probability of malignancy using dynamic breast F-18-FDG PET studies. J Nucl Med. 2009;50:1445. 
10. Visser EP, Philippens MEP, Kienhorst L, et al. Comparison of tumor volumes derived from glucose metabolic rate maps and SUV maps in dynamic 18F-FDG PET. J Nucl Med. 2008;49:892-898.

11. Sugawara Y, Zasadny KR, Grossman HB, Francis IR, Clarke MF, Wahl RL. Germ cell tumor: differentiation of viable tumor, mature teratoma, and necrotic tissue with FDG PET and kinetic modeling. Radiology. 1999;211:249-256.

12. Song S, Deng C, Wen L, et al. 18F-FDG PET/CT-related metabolic parameters and their value in early prediction of chemotherapy response in a VX2 tumor model. Nucl Med Biol. 2010;37:327-333.

13. Wang G, Parikh M, Nardo L, et al. Total-Body dynamic PET of metastatic cancer: first patient results. $J$ Nucl Med. 2020;61:208.

14. Chen K, Bandy D, Reiman E, et al. Noninvasive quantification of the cerebral metabolic rate for glucose using positron emission tomography, 18F-fluoro-2-deoxyglucose, the Patlak method, and an image-derived input function. J Cereb Blood Flow Metab. 1998;18:716-723.

15. Feng T, Tsui BMW, Li X, et al. Image-derived and arterial blood sampled input functions for quantitative PET imaging of the angiotensin II subtype 1 receptor in the kidney. Med Phys. 2015;42:6736-6744.

16. Rissanen E, Tuisku J, Luoto P, et al. Automated reference region extraction and population-based input function for brain [11C] TMSX PET image analyses. J Cereb Blood Flow Metab. 2015;35:157-165.

17. Yao S, Feng T, Zhao Y, et al. Simplified protocol for whole body Patlak parametric imaging with 18F-FDG PET/CT: feasibility and error analysis. Med Phys. 2020.

18. Karakatsanis NA, Lodge MA, Tahari AK, Zhou Y, Wahl RL, Rahmim A. Dynamic whole-body PET parametric imaging: I. Concept, acquisition protocol optimization and clinical application. Phys Med Biol. 2013;58:7391.

19. Zhang X, Xie Z, Berg E, et al. Total-body dynamic reconstruction and parametric imaging on the uEXPLORER. 
J Nucl Med. 2019;61:285-291.

20. Badawi RD, Shi $\mathrm{H}, \mathrm{Hu} \mathrm{P}$, et al. First human imaging study with the EXPLORER total-body PET scanner. $J$ Nucl Med. 2019:jnumed-119.

21. Yu D-C, Huang S-C, Barrio JR, Phelps ME. The assessment of the non-equilibrium effect in the'Patlak analysis' of Fdopa PET studies. Phys Med Biol. 1995;40:1243.

22. Feng T, Zhao Y, Shi H, et al. Total-Body quantitative parametric imaging of early kinetics of FDG. J Nucl Med. 2020:jnumed-119.

23. Lodge MA, Jacene HA, Pili R, Wahl RL. Reproducibility of tumor blood flow quantification with 15O-water PET. J Nucl Med. 2008;49:1620-1627.

24. Tsurusaki M, Okada M, Kuroda H, Matsuki M, Ishii K, Murakami T. Clinical application of 18F-fluorodeoxyglucose positron emission tomography for assessment and evaluation after therapy for malignant hepatic tumor. J Gastroenterol. 2014;49:46-56.

25. Wilson DW, Tsui BMW, Barrett HH. Noise properties of the EM algorithm. II. Monte Carlo simulations. Phys Med Biol. 1994;39:847.

26. Zhang X, Xie Z, Wang G, et al. Comparison of linear and non-linear total-body PET parametric imaging. J Nucl Med. 2020;61:206.

27. van Sluis J, Yaqub M, Brouwers AH, Dierckx RAJO, Noordzij W, Boellaard R. Use of population input functions for reduced scan duration whole-body Patlak 18 F-FDG PET imaging. EJNMMI Phys. 2021;8:1-8. 
TABLE 1. Dynamic frames for different protocols

\begin{tabular}{cccc}
\hline & Protocol 1 & Protocol 2 & Protocol 3 \\
\hline Start time $(\min )$ & 0 & 0 & 50 \\
& $5(\mathrm{~s}) * 30$ & $5(\mathrm{~s}) * 30$ & $120(\mathrm{~s}) * 3$ \\
Dynamic frames & $30(\mathrm{~s}) * 15$ & $30(\mathrm{~s}) * 3$ & $5(\mathrm{~s}) * 30$ \\
& $120(\mathrm{~s}) * 25$ & $50(\min ) * 1($ no scan $)$ & $30(\mathrm{~s}) * 3$ \\
\hline
\end{tabular}


TABLE 2, Patient data used in this study

\begin{tabular}{ccccl}
\hline Patient ID & Sex & Weight $(\mathrm{kg})$ & Injection dose $(\mathrm{MBq})$ & Preliminary Diagnosis \\
\hline 1 & M & 75 & 224.7 & Prostate cancer \\
2 & F & 60 & 223.5 & None \\
3 & F & 50 & 246.4 & Pulmonary nodule \\
4 & M & 60 & 317.1 & Space occupying lesion (brain) \\
5 & M & 83 & 306.0 & Gastric cancer \\
6 & F & 55 & 219.6 & Leiomyoma \\
7 & M & 81 & 375.7 & Pulmonary nodule \\
\hline
\end{tabular}



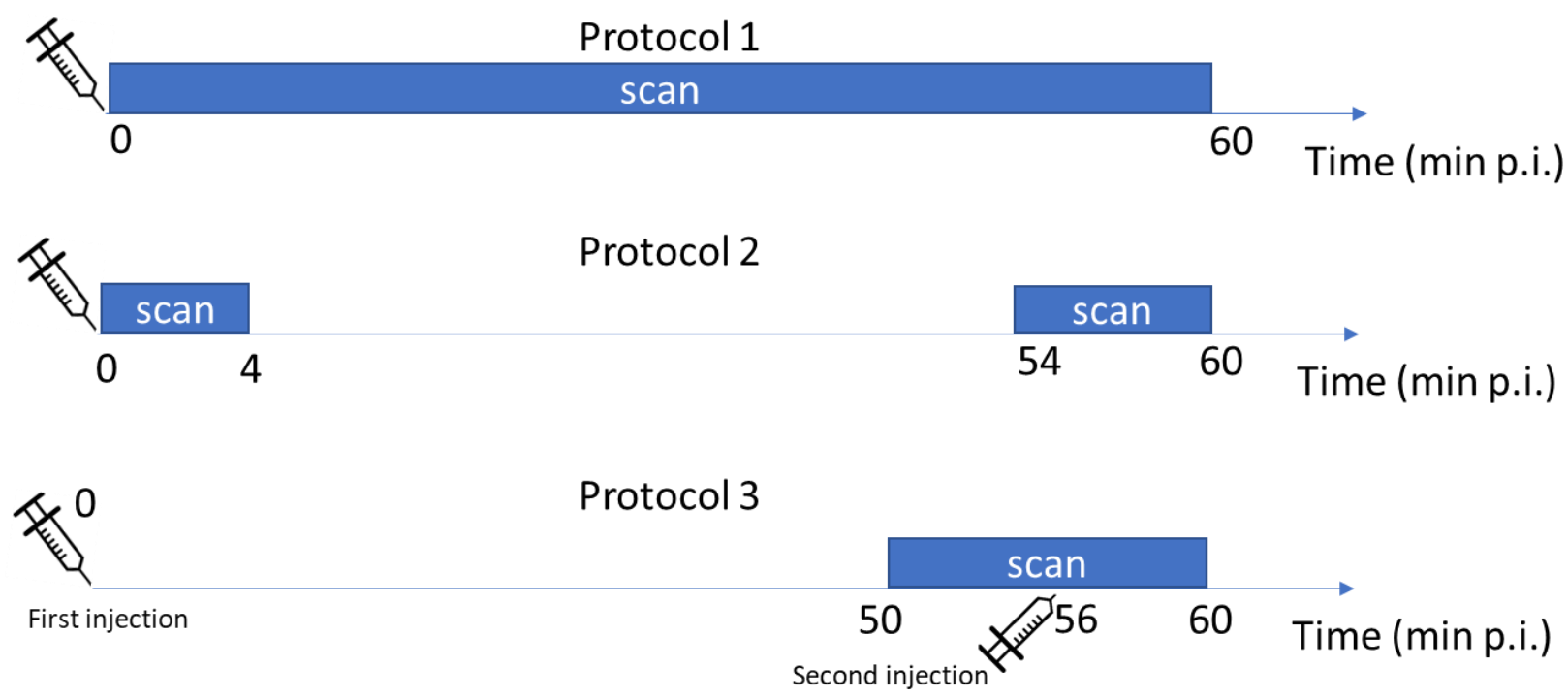

Figure 1. The illustration for the three protocols proposed in this study 

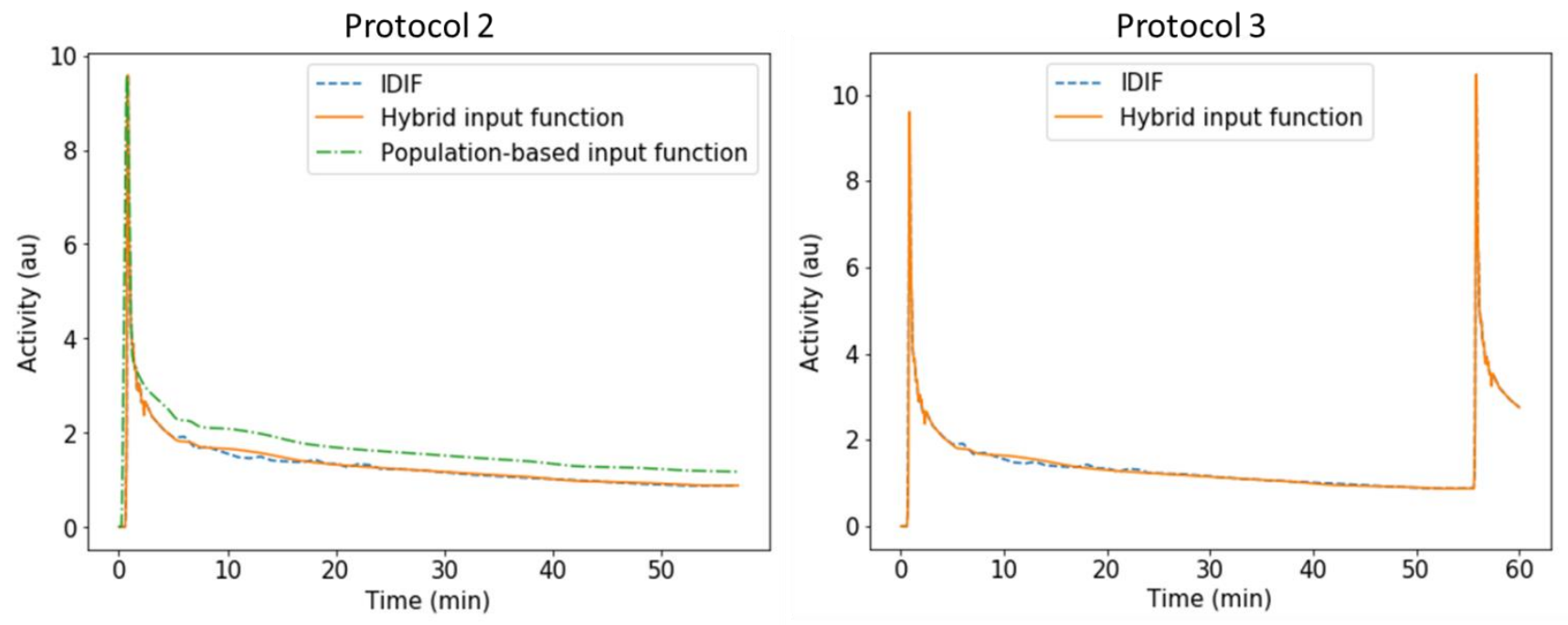

Figure 2 Comparison of the image-derived input function (IDIF) and the hybrid input function for Protocol 2 and Protocol 3. The original population-based input function was also displayed for comparison. 

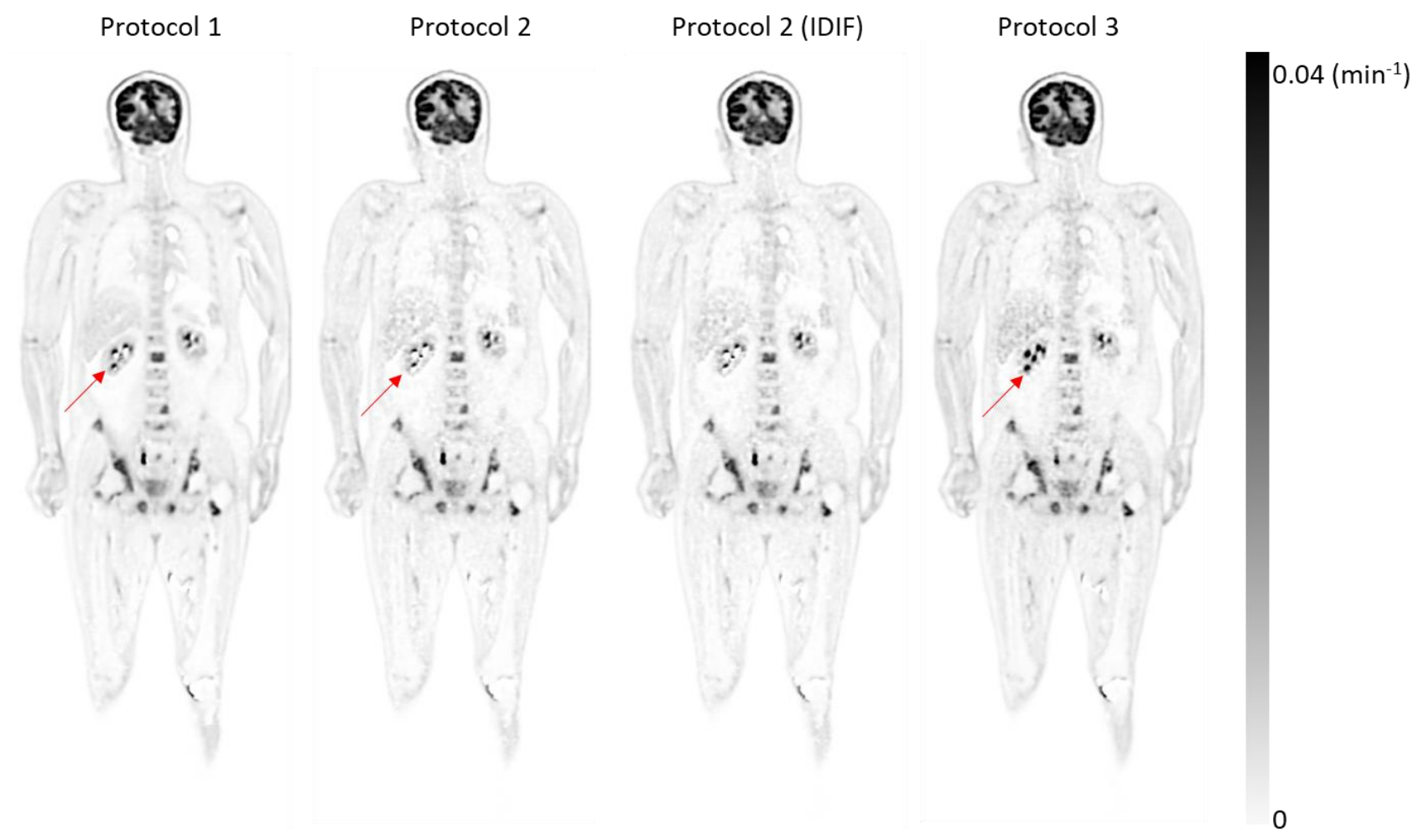

Figure 3 The estimated $K_{i}$ image of a patient with prostate cancer using Protocol 1, Protocol 2 with the hybrid input function, Protocol 2 with the image-derived input function, and Protocol 3 with the hybrid input function. Red arrows show regions with large $K_{i}$ differences using different protocols. 


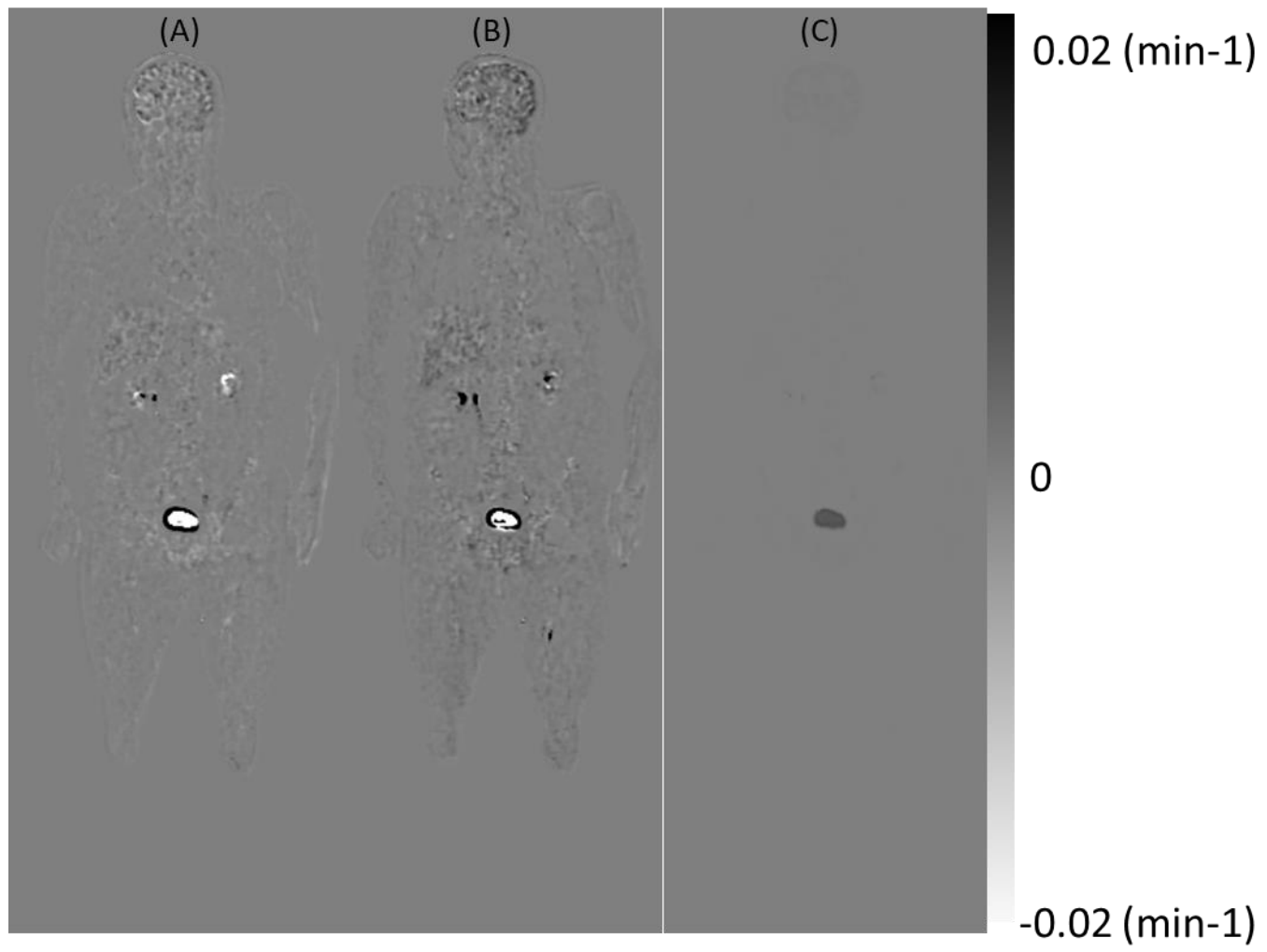

Figure 4 (A) The difference image of $K_{i}$ between Protocol 2 and Protocol 1. (B) The difference image of $K_{i}$ between Protocol 3 and Protocol 1. (C) The difference image of $K_{i}$ estimated using image-derived input function and the hybrid input function with Protocol 2 


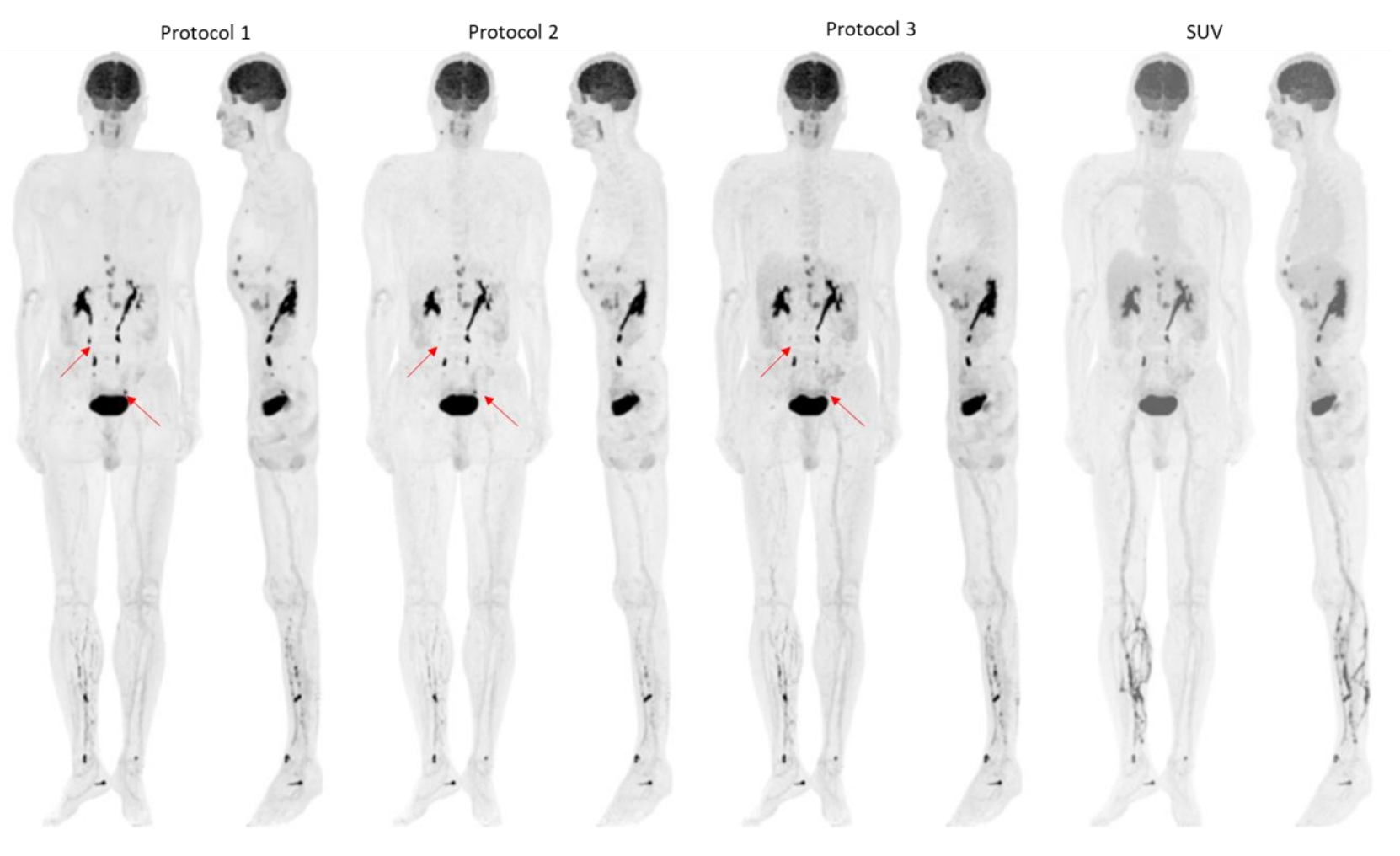

Figure 5. The maximum intensity projection image of $K_{i}$ from the three protocols and the SUV image acquired at $\mathrm{T}=60$ minutes. Red arrows show regions with large $K_{i}$ differences using different protocols. 


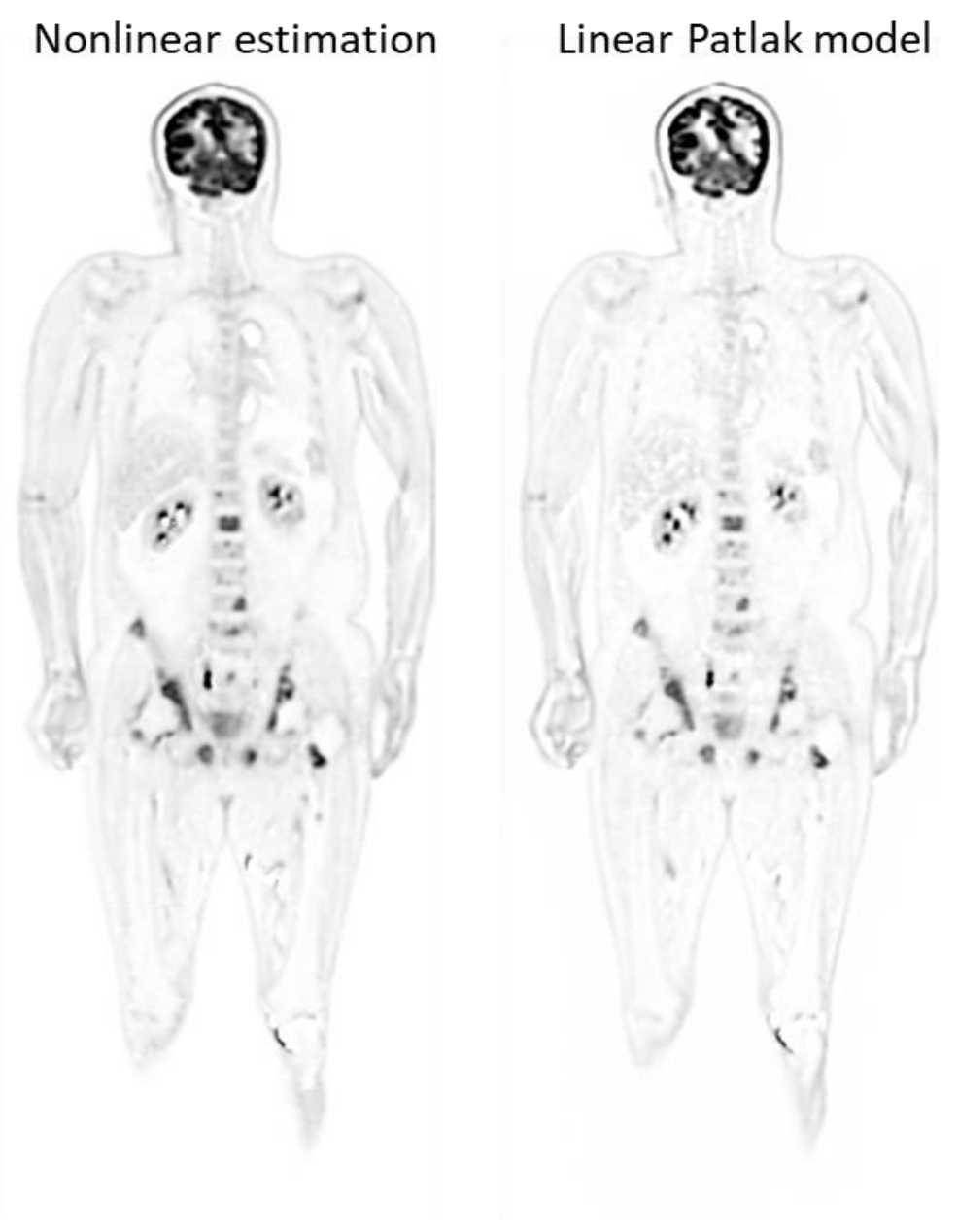

Figure 6. The estimated $K_{i}$ image using the nonlinear model (Protocol 1) and linear Patlak model. 

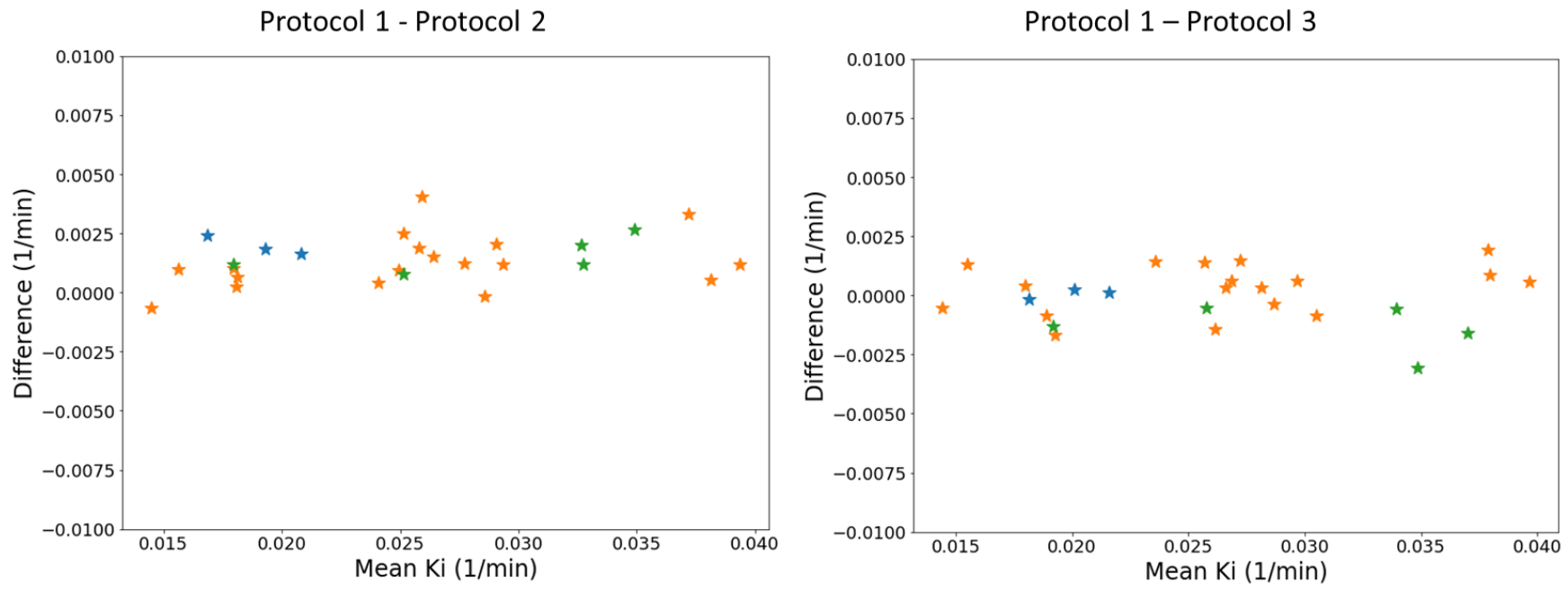

Figure. 7. The Bland-Altman plot for the estimated $\mathrm{K}_{\mathrm{i}}$ in different lesions using different protocols. $\mathrm{X}$-axis shows the mean $\mathrm{Ki}$ value and $\mathrm{y}$-axis shows the Ki difference. Lesions from different patients were encoded using different colors. 


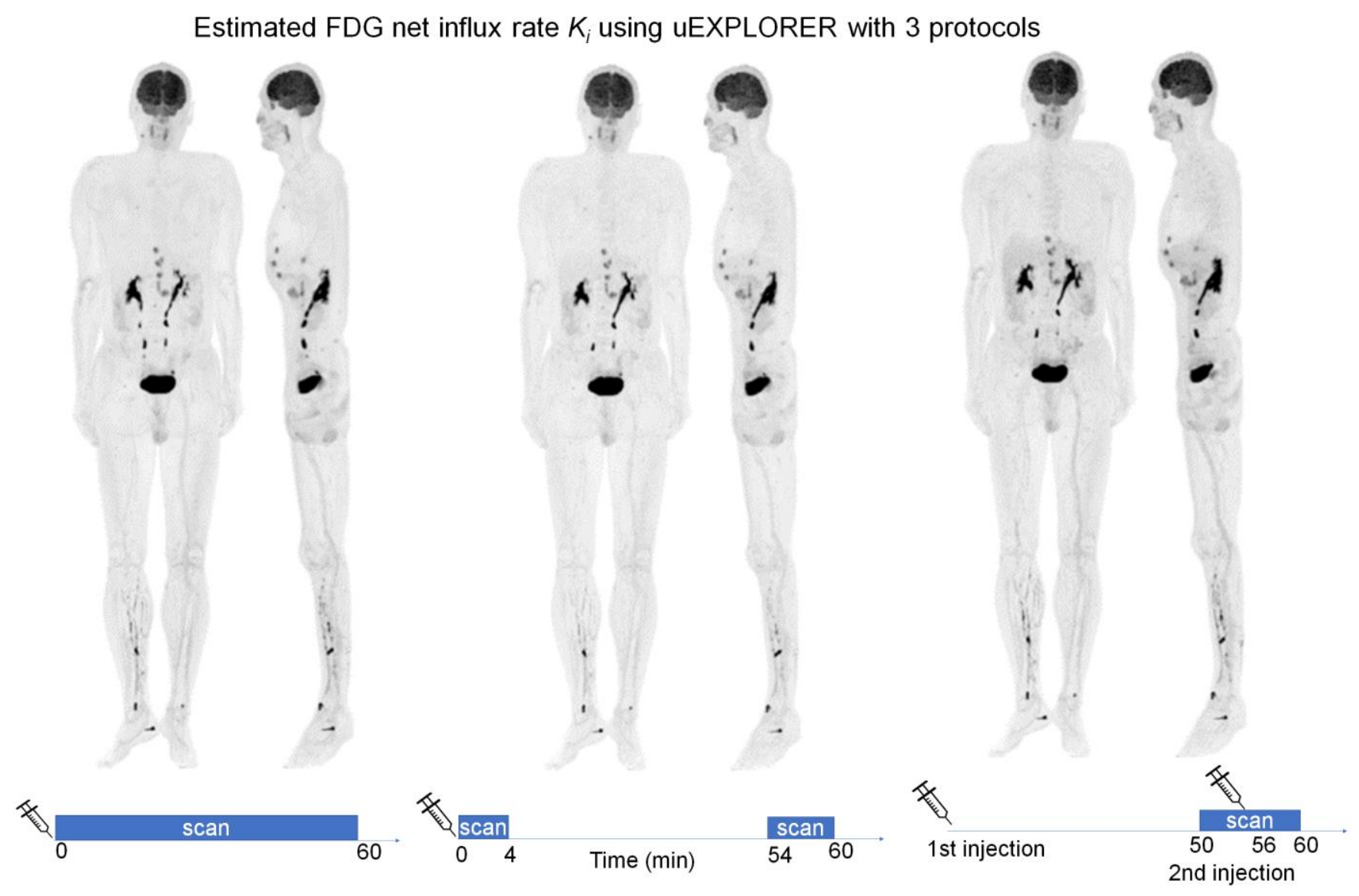


Supplemental data:
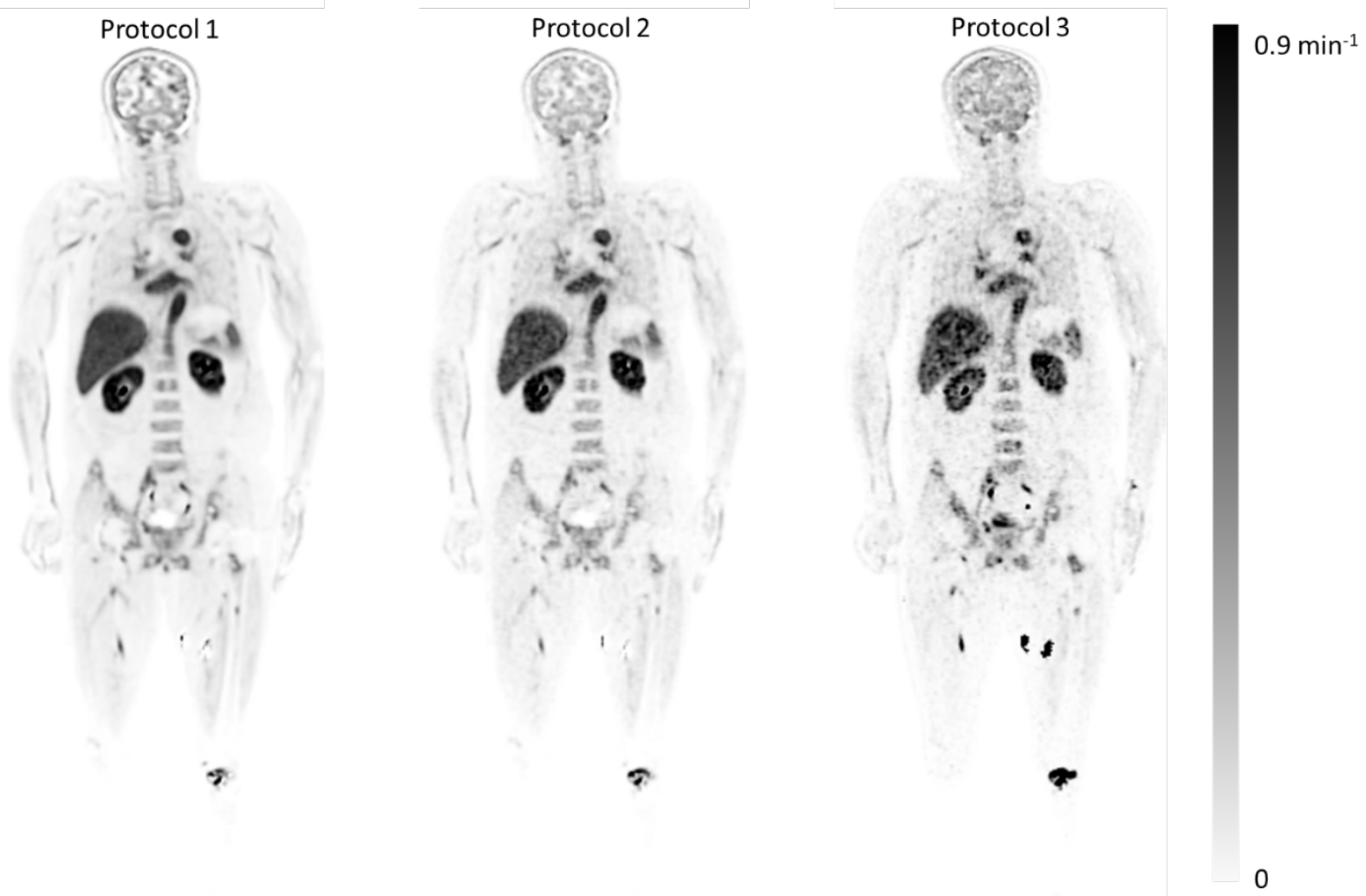

Supplemental Figure 1. $K_{l}$ ' images estimated using the three protocols. The three protocols in general shows similar K1' image. However, $K_{l}$ ' generated using Protocol 3 is significantly noisier. Some discrepancies around the bladder/urinary tract/leg vein regions. 


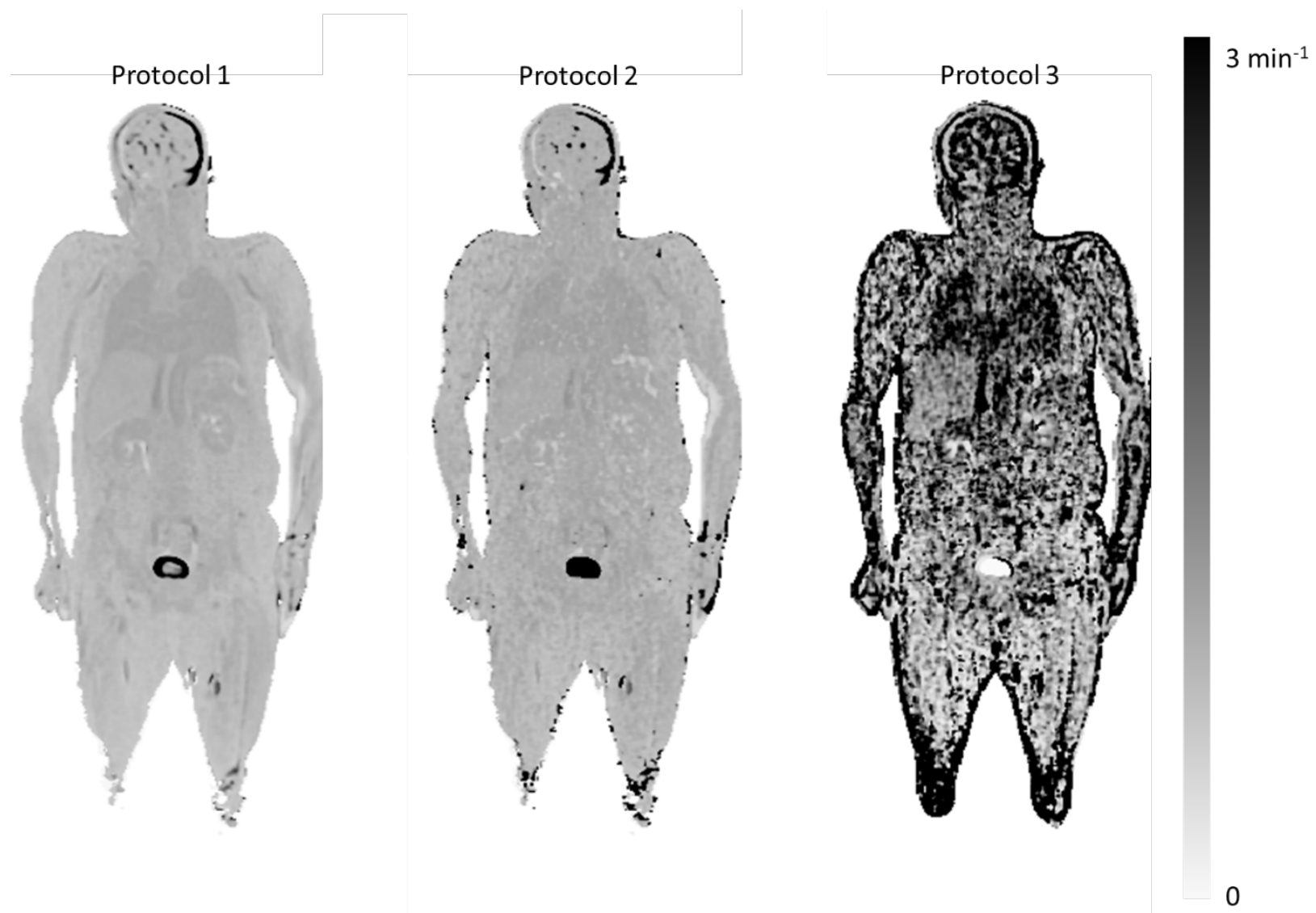

Supplemental Figure 2. $k_{2}$ ' images estimated using the three protocols.

The $k_{2}$ ' images estimate from Protocol 2 is visually comparable to that from Protocol 1, suggesting the combination of the early and the late information was sufficient for $k_{2}$ ' estimation. However, much higher pepper noise was present in the image generated using Protocol 3. suggesting that by adding the early and the late phase information in the same time periods, the estimation accuracy degrades. On the other hand, since most of the incorrect $k_{2}$ ' estimation is present in boundary locations or regions with small $K_{l}$ ', the impact of $k_{2}$ ' on $\mathrm{Ki}$ images is small and was found to cause minimal image artifacts. 


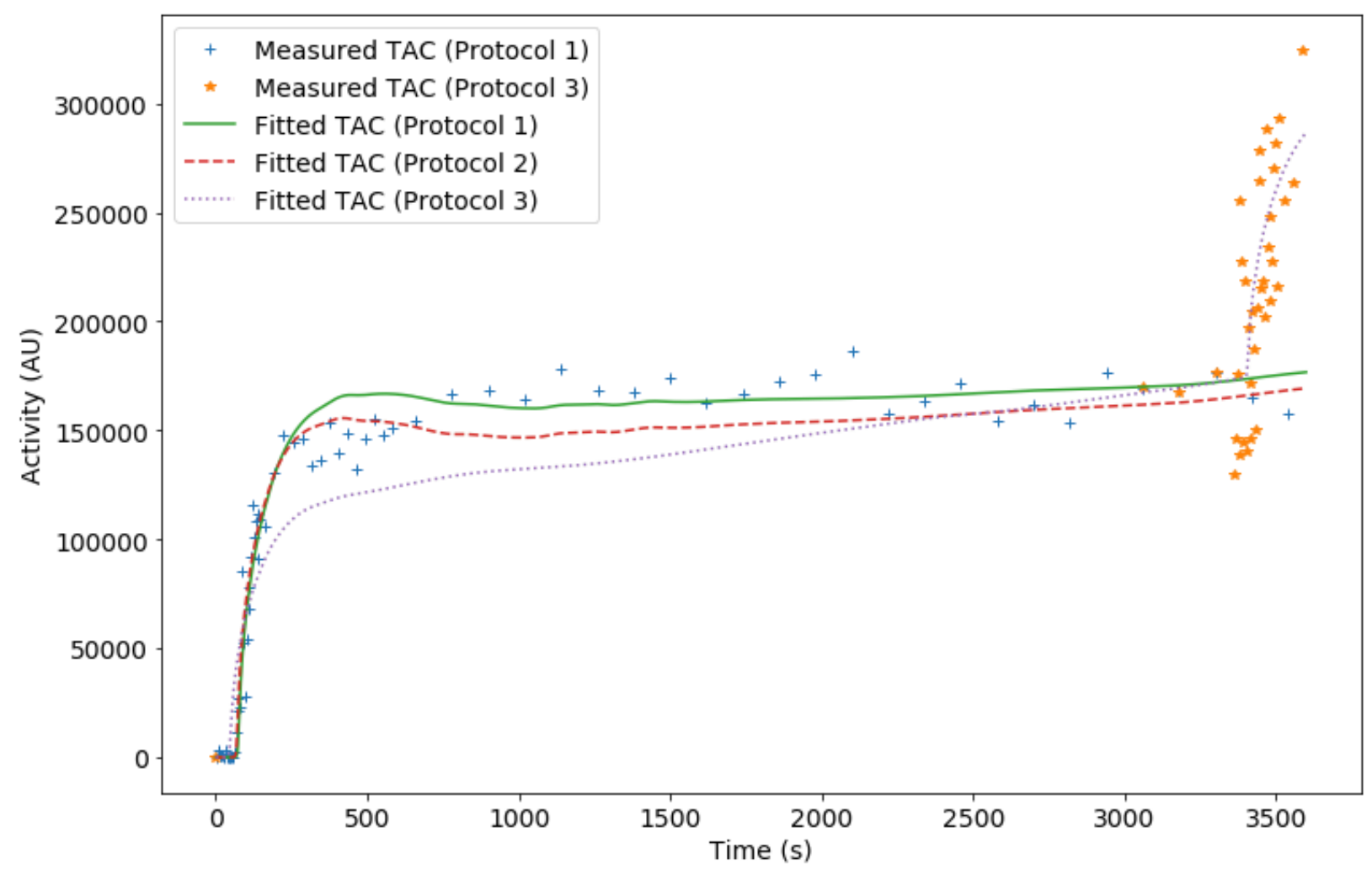

Supplemental Figure 3. Estimated TAC using the three Protocols for a lesion. For Protocol 2, only the early phase and the late phase of the measured TAC from Protocol 1 was used for fitting. For Protocol 3, only the data after 3000 seconds (orange stars) were used for fitting. 
Protocol 2-Protocol 1 Protocol 3-Protocol 1 IDIF - Hybrid input function

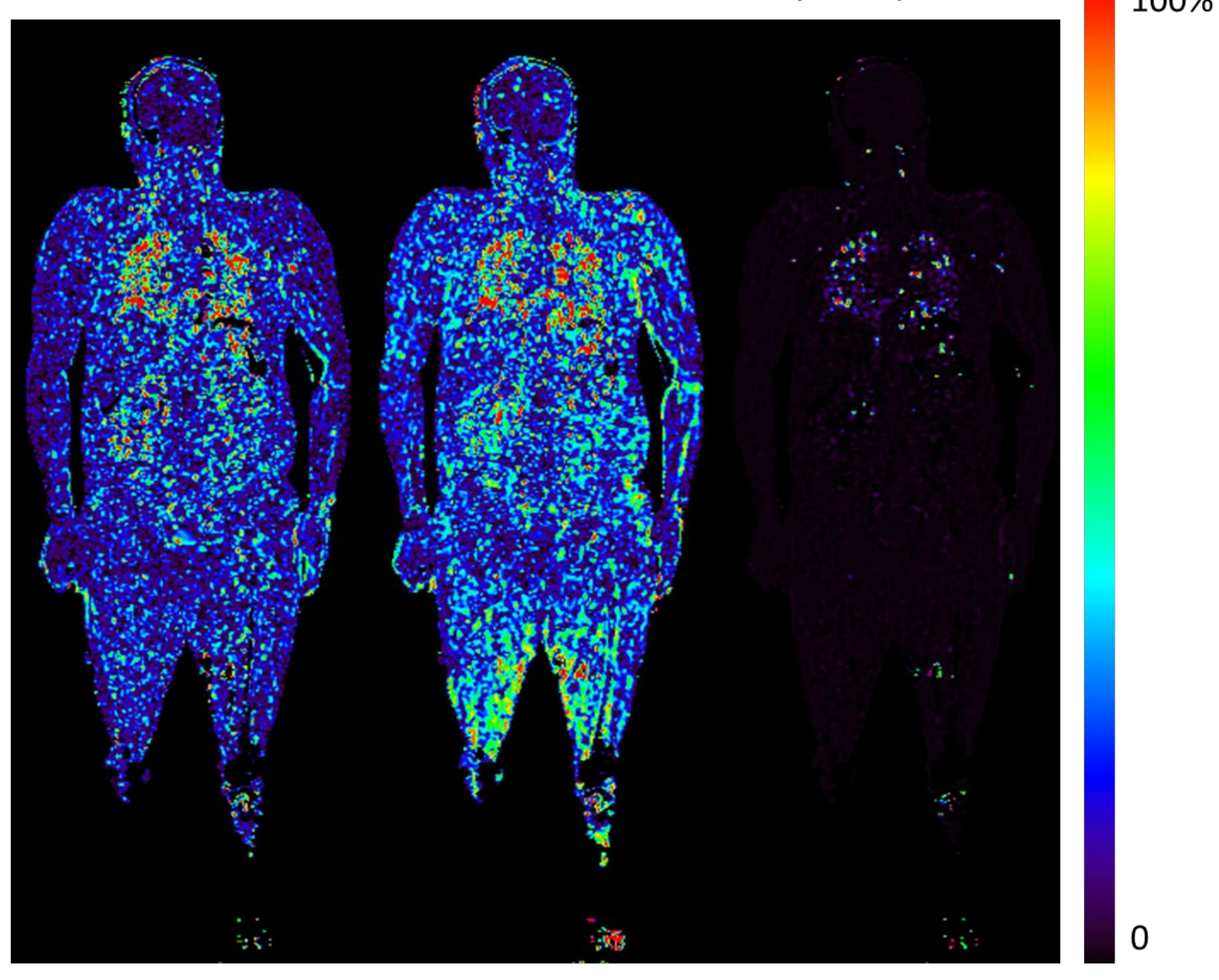

Supplemental Figure 4 The absolute percentage difference image of $K_{i}$ between Protocol 2 and Protocol 1 (left). The absolute percentage difference image of $K_{i}$ between Protocol 3 and Protocol 1 (middle). The absolute percentage difference image of $K_{i}$ estimated using image-derived input function and the hybrid input function with Protocol 2 (right) 